\title{
Human papillomavirus vaccine, teen sex and politics
}

\author{
Olufemi Olatunbosun
}

Infection with human papillomavirus (HPV) is a major risk factor for cervical cancer. The development of a safe and effective vaccine against HPV and its routine administration promises to be a major breakthrough in efforts at cervical cancer prevention. ${ }^{1}$ Research indicates that optimum benefit from the vaccine would be achieved in pre-adolescents ${ }^{2}$ to ensure protection against this sexually transmitted virus before sexual activity is initiated. The vaccine would probably be targeted primarily at girls but could also be used on boys to limit the spread of the virus, thereby providing an excellent example of male involvement in reproductive health. ${ }^{3}$ It is rather unfortunate that even before the vaccine becomes available commercially and introduced into service delivery, opposition is growing toward its widespread application. Conservative activists have begun posturing to influence how widely the immunisations will be employed. ${ }^{4}$ Religious groups in the USA are citing fears that giving the HPV vaccine could send a subtle message condoning premarital sex. ${ }^{5,6}$ They contend that vaccination could cause teens to feel that sexual activity is safer if they are vaccinated and may lead to risky sexual behaviour because they feel safe. They advocate provision of a better, healthier alternative than the vaccine, to avoid sabotaging sexual abstinence messages.

It is reassuring that research suggests that most parents are interested in having their pre-adolescent and adolescent children vaccinated against HPV. One survey showed that $80 \%$ of parents would endorse HPV vaccine for their preadolescent children. ${ }^{7}$ Still, it is disturbing to note that some health care providers would be less comfortable vaccinating younger versus older adolescents. ${ }^{8}$ Providers are concerned about potential negative reactions of parents to a recommendation of HPV vaccination. Endorsement of vaccination by professional organisations is of great importance. Parents value the information and recommendations provided by their children's health care providers. To that end, providers should set aside personal opinions and be guided by best practices and professional guidelines in the interest of their patients.

The provision of and widespread use of a vaccine that makes sex safer in children also faces political hurdles to funding by socially conservative governments around the world. In less developed countries where $80 \%$ of deaths from cervical cancer occur and where routine screening and treatment of precursors to cervical cancer are not available, ${ }^{9}$ one hopes that cost considerations and the need for three injections will not limit the use of HPV

J Fam Plann Reprod Health Care 2006; 32(2): 74

Department of Obstetrics, Gynecology and Reproductive Sciences, Royal University Hospital, University of Saskatchewan, Saskatoon, Saskatchewan, Canada Olufemi Olatunbosun, MD, FRCSC, Professor and Head of Department

Correspondence to: Prof. Olufemi Olatunbosun, Department of Obstetrics, Gynecology and Reproductive Sciences, Royal University Hospital, University of Saskatchewan, 103 Hospital Drive, Saskatoon, Saskatchewan S7N 0W8, Canada.

E-mail: olatunbosun@sask.usask.ca vaccine. In addition, social taboos may be even more powerful in developing countries and pose additional hurdles. The lessons learned from the resistance to polio vaccination in Northern Nigeria based on fear of AIDS and infertility 10 underscore the need for community education and involvement in policy decision-making regarding implementation of HPV vaccination.

Efforts at promoting acceptability of HPV vaccines should not be left to officials of the companies developing them, who have been trying to assuage the concerns of advocacy groups. Health care professional organisations need to formulate policies recommending widespread utilisation of potential HPV vaccines. It is reassuring that a recent survey of gynaecologists in the USA reported willingness to include this vaccine in their office practice. ${ }^{11}$ These findings should provide reassurance to those who are concerned about potential negative reactions of parents to a recommendation of HPV vaccination.

Recommending HPV vaccination of teens must be placed within the current context of efforts shifting toward the primary prevention of sexually transmitted infections through abstinence and postponing sexual activity. We encourage the adoption of comprehensive HPV prevention that includes routine vaccination yet does not compromise primary prevention efforts. A reproductive health approach with community participation and a client-centred focus (involving clients in programme design, implementation and evaluation) is essential to the successful introduction of this potentially useful vaccine.

Statements on funding and competing interests

Funding. None identified.

Competing interests. None identified.

References

1 Crum CP. The beginning of the end for cervical cancer? N Engl J Med 2002; 347: 1703-1705.

2 Mao C, Koutsky LA, Ault KA, Wheeler CM, Brown DR, Wiley DJ, et al. Efficacy of human papillomavirus-16 vaccine to prevent cervical intraepithelial neoplasia: a randomized controlled trial. Obstet Gynecol 2006; 107: 18-27.

3 Edouard L. Sex and the cervix: hormones, infections and cancer. $J$ Fam Plann Reprod Health Care 2005; 31: 338-339.

4 MacKenzie D. Will cancer vaccine get to all women? NewScientist.com news service, 18 April 2005. http://www. newscientist.com/channel/sex/mg18624954.500 [Accessed 9 February 2006].

5 Pollitt K. Virginity or death. The Nation, 30 May 2005. http://www.thenation.com/doc/20050530/pollitt [Accessed 9 February 2006].

6 Stein R. Debate rages on use of cervical cancer vaccine: while almost $100 \%$ effective, some contend use condones teen sex. San Francisco Chronicle, 31 October 2005. http://www.sfgate.com/cgibin/article.cgi?file=/c/a/2005/10/31/MNG2LFGJFT1.DTL [Accessed 9 February 2006].

7 Zimet GD. Parental attitudes about sexually transmitted infection vaccination for their adolescent children. Arch Pediatr Adolesc Med 2005; 159: 132-137.

8 Zimet GD. Improving adolescent health: focus on HPV vaccine acceptance. J Adolesc Health 2005; 37: S17-S23.

9 Sankaranarayanan R, Ferlay J. Worldwide burden of gynaecological cancer: the size of the problem. Best Pract Res Clin Obstet Gynaecol 2005 [Epub 12 December 2005].

10 Raufu A. Polio cases rise in Nigeria as vaccine is shunned for fear of AIDS. BMJ 2002; 324: 1414

11 Raley JC. Followwill KA, Zimet GD, Ault KA. Gynecologists' attitudes regarding human papilloma virus vaccination: a survey of Fellows of the American College of Obstetricians and Gynecologists. Infect Dis Obstet Gynecol 2004; 12: 127-133. 